\title{
Estudiantes afrodescendientes: desafíos en inclusión y \\ acompañamiento en la Universidad Santo Tomás, Bogotá*
}

[Artículos]

\author{
Manuela Preciado Cortés ${ }^{* *}$ \\ Adriana Riaño Triviño o***
}

Recibido: 4/11/202O

Aprobado: 15/o1/2021

Citar como:

Preciado, M. y Riaño, A. (2021). Estudiantes afrodescendientes: desafíos en inclusión y acompañamiento en la Universidad Santo Tomás, Bogotá. Análisis, 53(98).

https://doi.org/10.15332/21459169.6332

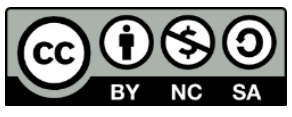

\section{Resumen}

El presente artículo presenta una revisión del proceso actual de inclusión a estudiantes afrodescendientes en la Universidad Santo Tomás (USTA), Bogotá. Se parte de la idea de que la dimensión cultural debe ser central

\footnotetext{
* El presente artículo de investigación hace parte de la convocatoria Jóvenes Investigadores USTA 2019 y se encuentra vinculado al proyecto de investigación "Nuevas perspectivas en acompañamiento estudiantil: comunidades de aprendizaje y TAC en la experiencia del Semillero Mareiwa", el cual hace parte de la convocatoria para el Fomento de la Investigación Fodein, 2019.

${ }_{* *}^{*}$ Estudiante de Derecho, Universidad Santo Tomás. Correo electrónico: manuelapreciado@usantotomas.edu.co

*** Psicóloga, por la Universidad Santo Tomás y magíster en Educación, por la Pontificia Universidad Javeriana. Docente de Humanidades de la Universidad Santo Tomás. Correo electrónico: adrianariano@usantotomas.edu.co; ORCID: https://orcid.org/0000-0002-4610-5196
} 
en la inclusión de estudiantes afrodescendientes para comprender su identidad, evitar prácticas de discriminación y racismo, y garantizar que la inclusión vaya más allá de beneficios económicos y que permita la incorporación de saberes de esta comunidad en las instituciones de educación superior (IES). Se implementó una metodología cualitativa, en la cual se revisaron documentos institucionales y se recolectaron testimonios a partir de entrevistas, que luego fueron canalizadas bajo las categorías de 'discriminación racial / racismo', 'identidad afrodescendiente' e 'inclusión'. Se encontró que, excepto por la política de descuentos, no hay, a la fecha, documentos ni prácticas orientadas explícitamente a la inclusión de la comunidad afrodescendiente en la USTA Bogotá; sin embargo, existe apertura desde las diferentes instancias para construirlas. Asimismo, en las entrevistas se evidenciaron diferencias respecto al concepto de 'afrodescendiente' y se enunciaron eventos de racismo que no contaron con ningún registro ni acompañamiento. Se concluye que se requiere un acuerdo institucional sobre lo que significa ser afrodescendiente y generar estrategias que vayan más allá de las cuotas o descuentos, y favorezcan la identificación y atención a las interacciones simbólicas que podrían producir escenarios de discriminación racial. Las políticas de inclusión para afrodescendientes deben responder tanto a las necesidades individuales como a la apertura de la Universidad para interactuar con el aporte histórico y cultural propio de esta comunidad.

Palabras clave: afrodescendientes, tutorías, inclusión, educación superior.

\section{Afro-descendant students: challenges in inclusion and tutoring in higher education- Universidad Santo Tomás, Bogota}

\section{Abstract}

This article presents a review of the current process of inclusion of Afrodescendant students at the Universidad Santo Tomás (USTA), Bogota. It 
is based on the idea that the cultural dimension must be cornerstone in the inclusion of Afro-descendant students to understand their identity, avoid discrimination and racism, and ensure that the inclusion goes beyond economic benefits and allows the incorporation of knowledge of this community in higher education institutions. A qualitative methodology was implemented, in which institutional documents were reviewed and testimonies were collected from interviews that were later channeled under the categories of 'racial discrimination / racism', 'Afrodescendant identity' and 'inclusion'. It was found that, except for the discount policy, to date there are no documents or practices explicitly oriented to the inclusion of the Afro-descendant community in USTA Bogota; however, there is openness from the different instances to build them. Likewise, the interviews revealed differences regarding the concept of 'Afro-descendant' and events of racism were voiced that did not have any record or support. It is concluded that there is a need for an institutional agreement on what means to be Afro-descendant and to generated strategies that go beyond quotas or discounts, and favor the identification and attention to symbolic interactions that could produce scenarios of racial discrimination. Inclusion policies for Afrodescendants should respond both to individual needs and to the openness of the University to interact with the historical and cultural contribution of this community.

Keywords: Afro-descendants, tutoring, inclusion, higher education.

\section{Estudantes afrodescendentes: desafios na inclusão e acompanhamento na Universidad de Santo Tomás, Bogotá}

\section{Resumo}

Este artigo apresenta uma revisão do atual processo de inclusão de estudantes afrodescendentes na Universidad Santo Tomás (USTA), Bogotá. Baseia-se na ideia de que a dimensão cultural deve ser central para a inclusão de estudantes de ascendência africana para entender sua 
identidade, evitar práticas de discriminação e racismo, e garantir que a inclusão ultrapasse os benefícios econômicos e permita a incorporação do conhecimento dessa comunidade nas instituições de ensino superior (IES). Foi implementada uma metodologia qualitativa, na qual foram revisados documentos institucionais e coletados depoimentos a partir de entrevistas, que foram então canalizadas nas categorias 'discriminação racial/racismo', 'identidade afrodescendente' e 'inclusão'. Verificou-se que, com exceção da política de descontos, não há, até o momento, documentos ou práticas explicitamente orientadas para a inclusão da comunidade afrodescendente na USTA Bogotá; no entanto, há abertura de diferentes instâncias para construí-los. Da mesma forma, as entrevistas evidenciaram diferenças em relação ao conceito de 'Afrodescendente' e expuseram eventos de racismo que não tinham registro ou acompanhamento. Conclui-se que é necessário um acordo institucional sobre o que significa ser afrodescendente e gerar estratégias que vão além de cotas ou descontos, e favorecer a identificação e a atenção às interações simbólicas que possam produzir cenários de discriminação racial. As políticas de inclusão para pessoas de ascendência africana devem responder tanto às necessidades individuais quanto à abertura da Universidade para interagir com a contribuição histórica e cultural própria dessa comunidade.

Palavras-chave: afrodescendentes, tutorias, inclusão, ensino superior.

\section{Introducción}

A medida que han avanzado los procesos de inclusión en las instituciones de educación superior (IES), se va profundizando la mirada sobre comunidades o grupos específicos. Uno de ellos es la comunidad afrodescendiente. En la USTA, al igual que en otras IES de Latinoamérica, se evidencia una primera etapa en esta apuesta por la inclusión, la cual trata de garantizar unos cupos de matrícula para estudiantes pertenecientes a esta comunidad. Sin embargo, esta acción inicial, si bien favorece el acceso y tiene un propósito de reparación histórica, no logra la 
inclusión completamente, pues existen aspectos culturales fundamentales que es necesario visibilizar para avanzar hacia una perspectiva más cercana a una nación plural, en la que se reconozca la igualdad de los pueblos y se visibilicen los aportes y liderazgos de las personas afrodescendientes.

Este texto abordará tres de los aspectos culturales que, de acuerdo con la literatura y los aportes de diferentes miembros de la comunidad educativa, se consideraron relevantes para tener una mejor comprensión de lo que podría ser el siguiente paso en inclusión para afrodescendientes. En un primer momento se abordará el binomio ‘discriminación racial/racismo', en el entendido de que estas prácticas implican la vulneración de derechos y, por lo mismo, son más evidentes y censurables, antecedidas por escenarios de discriminación racial que se dan en los intercambios culturales y que suelen ser menos evidentes y más sutiles. En segundo lugar, se abordará la identidad afrodescendiente, desde la comprensión de su complejidad más allá de las características fenotípicas. Por último, se abordará la inclusión y, dentro de ella, las acciones afirmativas, como estrategias de reconocimiento de derechos y resignificación cultural que favorecen los procesos de inclusión.

En el presente artículo, se analiza el caso de la Universidad Santo Tomás, Sede Bogotá; sin embargo, se presentarán algunos antecedentes en el marco jurídico que permiten, desde una perspectiva muy local, visibilizar algunos desafíos cruciales de la inclusión de estudiantes afrodescendientes en el panorama colombiano y realizar un breve contraste con las experiencias de las IES Latinoamericanas. Se pretende, entonces, afirmar que la dimensión cultural debe ser central en la inclusión de estudiantes afrodescendientes y tiene un papel relevante en el marco jurídico en relación con estos procesos, para comprender su identidad, evitar prácticas de discriminación y racismo, y garantizar que la inclusión vaya 
más allá de beneficios económicos, al igual que permita la incorporación de saberes de esta comunidad en las IES, con miras a favorecer la igualdad entre los pueblos.

\section{Discriminación racial y racismo}

En el interior de una sociedad, cuando el sector de la población considerado superior histórica y socialmente ejerce sobre una comunidad vulnerada actos de exclusión, vulneración de derechos y la restricción total o parcial en el goce y ejercicio de estos, por condiciones étnico-raciales y culturales propias e inherentes de la comunidad vulnerada, existe racismo y la discriminación racial (Segato, 2007).

Ahora bien, el racismo está compuesto por un factor interno y uno externo. El interno surge a partir de una actitud racista en el fuero íntimo de la persona, reflejado en sus convicciones personales; esto se denomina racismo prejuicio. Por otro lado, el factor externo, es la consecuencia del racismo prejuicio en la esfera pública, mediante el trato diferencialmente negativo y la vulneración de derechos; esto se denomina racismo discriminación (Segato, 2007).

El racismo prejuicio es un proceso cultural que se presenta en las interacciones cotidianas, comentarios, chistes, decisiones y no constituye en sí mismo alguna vulneración de derechos a la población sobre la cual recae. Sin embargo, este contexto antecede al racismo discriminación, en el cual sí se presenta la vulneración de algún derecho, y puede, por lo tanto, tener implicaciones jurídicas.

Bajo esta comprensión es necesario poner la mirada en el racismo estructural, comprendiendo que existen las prácticas y valores insertos en las instituciones, que promueven el estado de inferioridad de las personas 
discriminadas y el racismo, y reproducen las desventajas de la población discriminada.

Con lo anterior mente expuesto, es importante aclarar lo que se entiende por raza y etnia. La raza es una categoría histórica definida por la apariencia física o por el origen del grupo familiar; generalmente hace referencia a aspectos observables ya sea en la apariencia corporal (características fenotípicas) o en los documentos que permiten demostrar su parentesco. La etnia hace referencia a características culturales de la persona o comunidad que las vinculan a un grupo étnico en particular. Por ello que existen tres grupos sobre los cuales puede recaer el racismo: a) raza con etnicidad: tener características raciales y étnicas, es decir cuando un signo fenotípico se enlaza con un patrimonio cultural; b) raza sin etnicidad: cuando se tiene características raciales, pero no étnicas, es decir, cuenta con un signo fenotípico, pero no se vincula a un patrimonio cultural, y c) etnicidad sin raza: se cuenta con características étnicas, pero no raciales, es decir, se presenta el signo cultural pero no el fenotípico (Segato, 2007).

\section{Identidad afrodescendiente}

Las definiciones anteriormente presentadas nos llevan a precisar lo que se comprenderá en este documento como identidad afrodescendiente.

Organizaciones como Cepal entienden por afrodescendiente únicamente a los descendientes de africanos esclavizados en las Américas; sin embargo, dependiendo del país donde vivan estos descendientes, serán llamados con el prefijo afro y el nombre del país: "Cuando nos referimos a los afrodescendientes aludimos a las personas descendientes de africanos esclavizados en América Latina y el Caribe. Son también llamados 
"negros/as" y, según el país donde viven, se conocen por ejemplo como afroecuatorianos o afrocostarricenses” (Hopenhayn et ál., 2006).

El origen del término 'afrodescendiente' se establece en la Conferencia Regional de las Américas, contra el Racismo, la Discriminación Racial, la Xenofobia y las Formas Conexas de Intolerancia, celebrada en Santiago de Chile. Este concepto deja de lado el término 'negro' impuesto por los colonos y da paso al reconocimiento a un sujeto con resistencia política y con derechos. Por esto, el artículo hace referencia a la comunidad afrodescendiente y no "negra" o a "las negritudes". (Septien y Bidaseca, 2017).

El concepto de 'afrodescendencia' ha venido siendo determinado por hechos históricos como la esclavización de personas africanas en las Américas, factores fenotípicos, y elementos culturales originados únicamente por la esclavización. Estas aproximaciones, aunque válidas, promueven una definición que dista de la realidad actual, pues, en el presente, las personas con características fenotípicas o culturales afro pueden provenir también de personas africanas que no han vivido esclavitud. En ese sentido, aquí el término se extiende tanto a una persona descendiente de africanos esclavizados, como a migrantes africanos o migrantes con antecesores africanos.

\section{Inclusión}

En la actualidad el porcentaje de afrodescendientes en las IES corresponde al 12 \% en Latinoamérica (Freire et ál., 2018); sin embargo, la inclusión de esta población y el reconocimiento de su identidad y derechos aún presenta importantes desafíos para las comunidades educativas.

La inclusión de las diferentes poblaciones es uno de los retos actuales más relevantes en el sistema educativo, pues no solo las poblaciones diversas o 
tradicionalmente discriminadas merecen un trato digno y una educación ajustada a sus características, sino que también las comunidades educativas requieren de esa diversidad para construir comprensiones más amplias del mundo y de la propia identidad. La inclusión entonces implica un trabajo conjunto de la comunidad educativa.

Desde esta perspectiva, es importante resaltar:

Inclusión es un proceso. La educación inclusiva no es algo que tenga que ver meramente con facilitar el acceso a las escuelas ordinarias a los alumnos que han sido previamente excluidos. No es algo que tenga que ver con terminar con un sistema ordinario que no ha cambiado. El sistema escolar que conocemos -en términos de factores físicos, aspectos curriculares, expectativas, estilos del profesorado y roles directivos - tendrá que cambiar. Y ello porque la educación inclusiva es participación de todos los niños y jóvenes y remover, para conseguirlo, todas las prácticas excluyentes. (Arroyo, 2013, p. 150)

En ese sentido, para la misma la autora, se considera como

[...] el proceso de análisis sistemático de las culturas, las políticas y las prácticas escolares para tratar de eliminar o minimizar, a través de iniciativas sostenidas de mejora e innovación escolar, las barreras de distinto tipo que limitan la presencia, el aprendizaje y la participación de los alumnos y alumnas en la vida escolar de los centros donde son escolarizados, con particular atención a aquellos más vulnerables [...]. (Ainscow et ál., 2006, citado por Arroyo, 2013, p. 150)

Desde esta perspectiva, comprendemos que la inclusión no se limita al acceso, sino que tiene que ver con la participación (dar voz para entrar en diálogo) de las comunidades tradicionalmente excluidas y, por lo tanto, con construir transformaciones de las prácticas y los territorios. Esta definición de 'inclusión educativa' será la que oriente este estudio. 
Para el caso de la USTA Bogotá ${ }^{1}$, desde hace más de cinco años se cuenta con la Unidad de Desarrollo Integral Estudiantil (Udies), que tiene como propósito "promover, acompañar y articular programas que fortalezcan el ingreso, la participación, la permanencia y la graduación oportuna, mediante acciones coordinadas entre las distintas instancias académicas y administrativas a nivel nacional, que favorezcan el desarrollo" (Universidad Santo Tomás [USTA], 2015). Desde el periodo rectoral 20152018, esta unidad y la Unidad de Gestión Integral de la Calidad Universitaria (UGICU) desarrollan un proyecto de investigación que parte de las condiciones actuales de la USTA frente a las acciones incluyentes hacia las personas en condiciones diversas, para identificar los factores que pueden consolidar una política educativa, fundamentada en la identidad institucional, su historia y memoria, que se replica por todos los actores de la comunidad universitaria (USTA, 2018). Esta política será uno de los tópicos por explorar en relación con la inclusión de estudiantes afrodescendientes en la USTA Bogotá.

\section{Acciones afirmativas}

Ahora bien, uno de los procesos que materializa en mayor medida los procesos de inclusión de la población son las acciones afirmativas (AA), las cuales, según la Corte Constitucional, poyada en la Constitución Política de Colombia de 1991,

[...] fueron expresamente permitidas en la Carta para que el legislador pudiera, sin violar la igualdad, adoptar medidas en favor de ciertas

\footnotetext{
${ }^{1}$ La Universidad Santo Tomás (para este documento: USTA Colombia) es una Institución de Educación Superior católica de carácter privado, sin ánimo de lucro y de orden nacional. Hace presencia en cinco ciudades del país con sedes y seccionales en Bogotá, Bucaramanga, Medellín, Tunja y Villavicencio en la modalidad presencial y 23 Centros de Atención Universitaria CAU en la modalidad Abierta y a Distancia. La sede Principal Bogotá contaba, para 2019-1, con 21 programas y 9739 estudiantes de pregrado y 45 programas de posgrado.
} 
personas o grupos, sin tener que extender el beneficio resultante a otras personas o grupos que, por ello, se consideran discriminadas. [...] Cuando la Constitución protegió de manera especial a ciertos sujetos, permitió que sólo ellos fueran destinatarios de medidas específicas en su favor con el fin de avanzar hacia una sociedad menos desigual y un orden justo. (Constitución Política de Colombia, 1991, arts. 2 y 13)

Estas acciones provienen del ámbito jurídico, pues son prerrogativas creadas por el legislador. Por medio de ellas, una determinada comunidad o sector de la población, que por sus características particulares ha sido históricamente discriminada y excluida de diferentes sectores de la sociedad y en consecuencia ha sido vulnerable, puede acceder a espacios de los cuales ha sido excluida, ahora en condiciones menos desiguales. Las acciones afirmativas serían entonces, según Rita Segato,

[...] mecanismos para compensar y revertir formas de discriminación negativa que recayeron históricamente sobre las categorías sociales vulnerables como, por ejemplo, la población negra e indígena, en los diversos ámbitos de la vida social - política, jurídica, económica- y de las prácticas cotidianas. (2007, p. 82)

Para el caso de los estudiantes afrodescendientes, estas acciones se constituyen como medidas efectivas que aseguran que los miembros de comunidades étnicas históricamente discriminadas puedan acceder en condiciones de igualdad a una educación superior cuyo acceso ha sido preponderantemente para poblaciones hegemónicas que han ejercido una subyugación sobre las comunidades discriminadas. En este sentido, se presentan algunas experiencias de otras universidades latinoamericanas que vale la pena revisar. En la Universidad de la Frontera en Chile (Ufro), el $13 \%$ de la comunidad estudiantil pertenece a la comunidad indígena mapuche; allí se implementaron acciones afirmativas para este grupo, orientadas, en un primer momento, a generar conocimiento sobre la 
situación de la población estudiantil mapuche en la Ufro y determinar la existencia de AA existentes allí; y luego la elaboración, aplicación y evaluación del programa de apoyo académico para estudiantes mapuches. El proceso, que duró cerca de 5 años, pretendió documentar la experiencia con el fin de difundirla y estimular la adopción de AA (Universidad de La Frontera, 2019).

El caso de Perú es un caso particular, pues dentro del desarrollo y aplicación de las acciones afirmativas, se constituyeron dos universidades indígenas, además de otras bilingües en las que se imparten clases en castellano y determinado dialecto indígena (Sanborn y Arrieta, 2011). En Brasil, la creación y aplicación de acciones afirmativas en las instituciones educativas superiores, se dio por la presión de los medios de comunicación y algunas luchas políticas. Este es el caso de la Universidad del Estado de Río de Janeiro, la cual implementó una prueba de ingreso, con un sistema de cuotas mínimas para estudiantes autodeclarados negros (Hermida, 2007).

\section{Contexto jurídico colombiano}

En Colombia, la Ley 70 de 1993 es la fuente jurídica con mayor relevancia para la comunidad afrodescendiente, dado que es la única herramienta jurídica específica para esta comunidad; se desprende de la Constitución de 1991 y brinda el marco para el aseguramiento de derechos. Las directrices presentadas en dicha ley se materializan en casos concretos a partir de sentencias que marcan hitos y orientaciones para avanzar en la reivindicación de los derechos de esta comunidad. Algunas de estas sentencias están relacionadas con acciones afirmativas para la población afrodescendiente del territorio colombiano y tienen una gran significación, ya que, al ser la comunidad afro marginada, discriminada e invisibilizada a través de la historia con huellas vigentes, es importante establecer 
mecanismos que le permitan participar dentro de la sociedad de forma activa y equitativa.

La Corte Constitucional, en la sentencia T-68o/16, destacó la importancia de las acciones afirmativas en el ámbito educativo para la comunidad afrodescendiente. De no ser aplicadas, se estarían violentando otros derechos y garantías fundamentales de la comunidad y de la persona en general:

En conclusión, la Constitución, el Bloque de Constitucionalidad, la ley y los reglamentos internos de las universidades, públicas y privadas, reconocen la necesidad de crear medidas afirmativas que acerquen a los miembros de las comunidades negras a escenarios de igualdad efectiva en materia de educación superior, logrando así que una comunidad clásicamente discriminada acceda en condiciones dignas a una prestación esencial para garantizar otros derechos. Por lo anterior, negarle el acceso a una persona miembro de estas comunidades a una medida de discriminación positiva de la que son sujeto implica la vulneración de su derecho a la igualdad ${ }^{2}$. (Sentencia T-680/16, 2016)

Conforme a lo anterior, en la Universidad del Valle, de Cali, se desarrollaron acciones afirmativas por medio de "cupos especiales" para las comunidades afrodescendientes:

En el año 2003 mediante la Resolución 097/03, el Consejo Académico de la Universidad del Valle, aprobó el 4 \% del cupo de cada programa académico para aspirantes pertenecientes a las comunidades negras. Posteriormente, el Consejo Superior aprobó la Resolución n. ${ }^{\circ} 038$ del 13 de mayo de 2010, a través de la que la Universidad del Valle establece "la exención en el valor de la matrícula básica de los Programas Académicos

2 Énfasis de las autoras. 
de Pregrado denominada "Comunidades Negras o Afrocolombianas".

(Universidad del Valle, Resolución n. ${ }^{\circ}$ 097, 2003, p. 4)

Por su lado, la región Caribe colombiana expone cuáles fueron las políticas de inclusión desarrolladas por siete IES públicas de la región. Dichas políticas o mecanismos de inclusión son denominadas "apoyos", los cuales pueden ser financieros, académicos y otros, además de "cupos especiales" asignados a los estudiantes matriculados de poblaciones vulnerables. (Morón-Cárdenas et ál., 2013).

\section{Tres sentencias, tres formas de materializar las acciones afirmativas}

Tanto la legislación vigente como las sentencias presentadas en este artículo exponen el fenómeno de la discriminación, que ha sido impedimento para el acceso de forma equitativa e igualitaria al ejercicio de los derechos y acceso a las garantías de las comunidades que la sufren. También dan las herramientas pertinentes para poder ser empleadas y aplicadas tanto por el sector de la sociedad que puede llegar a ejercer la discriminación como por el sector que la recibe y, por medio de ello, se pretende llegar al acceso pleno de los derechos y garantías, sin impedimentos por razón de etnia, rasgos fenotípicos o condición específica.

No obstante, a pesar de que se ha venido abordando la acción afirmativa o política de inclusión desde un enfoque académico y destinado para las comunidades étnicas, la Corte Constitucional, en reiteradas ocasiones y diferentes circunstancias, nos ha permitido comprender que estas fueron creadas para todo sector de la población que se considere vulnerable por parte del legislador.

En la sentencia C-964/o3 de la Corte Constitucional se aborda la discriminación positiva, en torno a la formación de igualdad de género. 
Con ella, para darle sentido a esta condición, el legislador dispuso prerrogativas para la mujer cabeza de familia, excluyendo a los hombres con la misma condición, debido a la discriminación histórica de la que ha sido víctima la mujer. Además de ello, es importante resaltar que la misma Corte expone que dichas prerrogativas no se pueden extender a otros sujetos. De acuerdo con esto, se podría establecer que, para poder acceder a las acciones afirmativas, es indispensable ser parte de una población considerada históricamente vulnerable, pues si hay sujetos con la misma situación fáctica, pero sin contar con tal calidad, no podría acceder a ellas invocando aplicación de igualdad. Finalmente, dicha igualdad no se le puede conceder en aras de formar una sociedad menos desigual.

Las llamadas acciones afirmativas fueron expresamente permitidas en la Carta para que el legislador pudiera, sin violar la igualdad, adoptar medidas en favor de ciertas personas o grupos, sin tener que extender el beneficio resultante a otras personas o grupos que, por ello, se consideran discriminadas [...]. Cuando la Constitución protegió de manera especial a ciertos sujetos, permitió que solo ellos fueran destinatarios de medidas específicas en su favor con el fin de avanzar hacia una sociedad menos desigual y un orden justo. (Corte Constitucional, Sentencia C-964/03, 2003)

Por otro lado, la sentencia C-293/10 desarrolla el concepto de discriminación positiva alrededor de las personas con algún tipo de discapacidad que les impida poder acceder a determinados sectores laborales, económicos y sociales de la colectividad. Lo interesante de esta sentencia es que presenta el origen de las acciones afirmativas, las cuales surgen en los Estados Unidos en el siglo XX para salvaguardar a la comunidad negra de los escenarios de discriminación de los cuales han sido sujetos durante años. Asimismo, plantea que, detrás de cada acción 
afirmativa, hay un precio y una obligación que debe ser asumida en conjunto por la sociedad, procurando que dicha carga no sea desmedida:

En todos los casos la implementación de una acción afirmativa conlleva costos o cargas, que deben ser razonables, y que frecuentemente se diseminan y son asumidos por la sociedad como conjunto. Sin embargo, debe resaltarse que, en el caso de las acciones de discriminación positiva, la carga puede recaer de manera exclusiva sobre personas determinadas. (Corte Constitucional, Sentencia C-293/10, 2010)

Finalmente, en la sentencia C-115-2017, se busca proteger el derecho constitucional al trabajo, por medio de exenciones establecidas específicamente para la población juvenil, con la finalidad de que pueda acceder a un empleo. Demuestra la Corte que está en la búsqueda de crear condiciones donde la protagonista sea la igualdad materializada. Sin embargo, la expresa que estas medidas también traen consigo consecuencias positivas para la macroeconomía, aportes tributarios y al sistema de seguridad social y formalización del empleo. Esta situación pone una alerta sobre la posibilidad de que las acciones afirmativas puedan, en algunos casos, apoyarse en la garantía de igualdad, pero persigan intereses que terminan privilegiando a sectores que tradicionalmente han oprimido a las mismas poblaciones que se busca proteger.

La sentencia T-680/16 concluyó que el propósito de la creación de medidas afirmativas dirigidas hacia la comunidad afrodescendiente tiene el objetivo de acercar a dichas comunidades a escenarios de igualdad en materia de educación superior. Asimismo, el negar el acceso de una acción afirmativa a un miembro de esta comunidad implica la vulneración de su derecho a la igualdad. 
En este sentido, la USTA ha realizado un avance en la creación de acciones afirmativas, teniendo en cuenta la revisión de documentos institucionales. Estas acciones se ven reflejadas en un descuento en la matrícula, que aunque es útil, podría tener un mayor impacto si se amplía la mirada, dada la realidad que vive la comunidad afro en la institución, así como las barreras para acceder y permanecer en la Universidad en condiciones de igualdad. Un verdadero avance consistiría en incluir el componente étnico de la comunidad, que es indispensable para entender cuáles pueden ser las herramientas que la USTA puede intentar implementar, de modo que dichas acciones sean realmente efectivas y abarquen las realidades de una comunidad clásicamente discriminada y protejan su derecho a la igualdad.

En el caso concreto, por medio de instrumentos jurídicos como las sentencias, los sectores de la sociedad clásicamente discriminados exponen al Estado sus principales problemáticas, y es el Estado por medio de las altas cortes el que manifiesta su postura y reconoce que se han vulnerado los derechos de estos sectores de la población de forma constante en el tiempo. Así, por medio de las decisiones que allí manifiesta el Estado, despliega una serie de acciones que está obligada a cumplir la sociedad en general para detener la vulneración de derechos de las comunidades discriminadas. Justamente, la construcción de acciones afirmativas, aterrizadas en los diferentes sectores de la sociedad, dará lugar a un acceso igualitario y equitativo al goce y ejercicio de los derechos de las comunidades que las necesitan.

\section{Método}

Dado que la pretensión inicial de este estudio es tener una comprensión de la situación actual de la inclusión para estudiantes afrodescendientes en la USTA Bogotá, se ha optado por una metodología de triangulación. La triangulación se refiere al uso de varios métodos (tanto cuantitativos como 
cualitativos), de fuentes de datos, de teorías, de investigadores o de ambientes en el estudio de un fenómeno. La triangulación ofrece una oportunidad para que se elabore una perspectiva más amplia en cuanto a la interpretación del fenómeno en cuestión, porque señala su complejidad; esto, a su vez, enriquece el estudio y brinda la oportunidad de que se realicen nuevos planteamientos (Benavides y Gómez-Restrepo, 2005, p. 118).

La triangulación es relevante para este tipo de estudios, dado que ha sido hasta ahora un tema poco explorado en la USTA Bogotá y es importante comprenderlo a partir de distintas fuentes que permiten obtener un panorama más completo de la situación el cual involucre distintas miradas al mismo fenómeno.

En este sentido, se recogieron tres tipos de datos diferentes para comprender la situación de los estudiantes afrodescendientes en la USTA Bogotá. Inicialmente se acudió a la revisión de tres documentos institucionales para conocer: a) cómo la Universidad actúa respecto a la inclusión de estudiantes afrodescendientes; b) si existen algunas decisiones institucionales, y c) en qué oportunidades se menciona a esta comunidad dentro de dichos textos. En segunda instancia, se recurre a la base de datos del sistema académico SAC, con el fin de tener un panorama general respecto a la cantidad de estudiantes afrodescendientes matriculados en relación con el total de estudiantes. Por último, para poder ampliar la comprensión a partir de los testimonios de diferentes personas relacionadas con el proceso de inclusión de estudiantes afrodescendientes, se realizan seis entrevistas: cuatro estudiantes afrodescendientes cuya selección estuvo basada en un muestreo por conveniencia, dada la imposibilidad de conocer el listado de estudiantes identificado como "negritudes", y la cercanía y disponibilidad de los estudiantes con una de las autoras del presente estudio, también 
afrodescendiente; un docente no afrodescendiente, y un administrativo no

afrodescendiente, quienes fueron seleccionados por su relevancia y

trayectoria en la Universidad en los procesos de inclusión y su

participación directa en la construcción de las políticas de inclusión de la

Universidad.

En la tabla 1 se describen las fases en las que se realizó el estudio:

Tabla 1. Ruta del estudio

\begin{tabular}{|c|c|c|c|}
\hline $\begin{array}{c}\text { Fases del } \\
\text { proceso }\end{array}$ & Tiempo & Objetivo & Fuentes \\
\hline $\begin{array}{l}\text { Fase } 1 . \\
\text { Recolección de } \\
\text { datos } \\
\text { institucionales }\end{array}$ & $\begin{array}{l}\text { Primer } \\
\text { semestre, } \\
2015^{-} \\
\text {segundo } \\
\text { semestre, } \\
2018\end{array}$ & $\begin{array}{l}\text { Identificar la mención de } \\
\text { la población } \\
\text { afrodescendiente en tres } \\
\text { documentos } \\
\text { institucionales. } \\
\text { Identificar la forma de } \\
\text { registro de los estudiantes } \\
\text { afrodescendientes y la } \\
\text { cantidad de inscritos en } \\
\text { relación con la cantidad } \\
\text { total de estudiantes de } \\
\text { pregrado, Bogotá. }\end{array}$ & $\begin{array}{l}\text { Documento marco de } \\
\text { Desarrollo Integral del } \\
\text { estudiante (2015), } \\
\text { Reglamento de auxilios y } \\
\text { descuentos educativos } \\
\text { (2019) y el Informe de } \\
\text { gestión - Rectoría General } \\
\text { 2015-2018 (2018). } \\
\text { Base de datos de SAC 2015- } \\
\text { 2018, registro de inscritos } \\
\text { que se han identificado como } \\
\text { "Negritudes". }\end{array}$ \\
\hline $\begin{array}{l}\text { Fase } 2 . \\
\text { Elaboración de } \\
\text { entrevistas } \\
\text { semiestructuradas }\end{array}$ & $\begin{array}{l}\text { Junio - } \\
\text { agosto, } 2019\end{array}$ & $\begin{array}{l}\text { Comprender las } \\
\text { perspectivas de diferentes } \\
\text { actores de la comunidad } \\
\text { educativa en relación con } \\
\text { la inclusión de estudiantes } \\
\text { afrodescendientes. }\end{array}$ & $\begin{array}{l}\text { Entrevistas realizadas a } \\
\text { cuatro estudiantes } \\
\text { afrodescendientes, un } \\
\text { docente y una directiva (líder } \\
\text { de la Udies), vinculados con } \\
\text { la construcción de las } \\
\text { políticas de inclusión. }\end{array}$ \\
\hline $\begin{array}{l}\text { Fase } 3 . \\
\text { Sistematización } \\
\text { de los datos }\end{array}$ & $\begin{array}{l}\text { Septiembre - } \\
\text { noviembre, } \\
2019\end{array}$ & $\begin{array}{l}\text { Organizar los datos de } \\
\text { acuerdo con las categorías } \\
\text { definidas: 'discriminación } \\
\text { racial / racismo'; } \\
\text { 'identidad } \\
\text { afrodescendiente'; } \\
\text { 'inclusión' (acciones } \\
\text { afirmativas). }\end{array}$ & $\begin{array}{l}\text { Se utilizó el software } \\
\text { oTranscribe, para la } \\
\text { transcripción de las } \\
\text { entrevistas, y el software } \\
\text { Excel tanto para la } \\
\text { categorización de las } \\
\text { entrevistas como para el } \\
\text { análisis de la base de datos } \\
\text { de SAC. }\end{array}$ \\
\hline $\begin{array}{l}\text { Fase 4. Análisis de } \\
\text { los datos }\end{array}$ & $\begin{array}{l}\text { Noviembre, } \\
2019\end{array}$ & $\begin{array}{l}\text { Interpretar la información } \\
\text { sistematizada con el fin de } \\
\text { comprender la situación } \\
\text { de los estudiantes } \\
\text { afrodescendientes en la } \\
\text { USTA, a partir de las } \\
\text { categorías definidas. }\end{array}$ & $\mathrm{N} / \mathrm{A}$ \\
\hline
\end{tabular}

Fuente: elaboración propia. 


\section{Resultados}

La presentación de los resultados hallados en cada fuente, se realizará a partir de las tres categorías de análisis propuestas en la introducción: discriminación racial / racismo; identidad afrodescendiente; inclusión (acciones afirmativas).

\section{Identidad afrodescendiente}

Respecto al registro de estudiantes afrodescendientes, de acuerdo con la información suministrada por el Sistema Académico SAC, entre los años de 2015 y 2018, 44 estudiantes de 82728 fueron registrados en el sistema con caracterización "Negritudes" en ante la USTA, es decir un 0,053 \%. En la tabla 2 se presenta la relación por semestre y programa académico:

Como se evidencia, el nivel de caracterización de los estudiantes es muy bajo $\mathrm{y}$, si bien no hay un registro alternativo que nos permita identificar si existe un subregistro, es importante preguntarse por este aspecto, que puede tener dos explicaciones: por un lado, el hecho de que los estudiantes ven como no relevante o incluso vergonzoso identificarse como estudiantes afrodescendientes o que, tal como lo manifestó uno de los estudiantes participantes:

[...] algunos estudiantes afrodescendientes solo se identifican como tal con el propósito de obtener el descuento en la matrícula, pues no hay ningún otro beneficio, pero, para obtener ese descuento es necesario presentar un certificado que muchas veces cuesta igual que lo que le van a descontar a uno, entonces uno no lo saca ni solicita el descuento. (Estudiante 1, comunicación personal, octubre de 2019) 
Asimismo, es relevante revisar el registro en el SAC, debido a que la categoría "Negritudes" no integra completamente la identidad afrodescendiente, pues este término se limita a la característica fenotípica y desconoce los componentes étnicos que constituyen ser afro. 
Tabla 2. Estudiantes con caracterización "Negritudes" en el sistema académico SAC

\begin{tabular}{|c|c|c|c|c|c|c|c|c|}
\hline Etiquetas de fila & $2015-1$ & 2015-2 & 2016-1 & 2016-2 & 2017-2 & 2018-2 & 2018-1 & Total general \\
\hline Administración de empresas & 1 & & 1 & & 2 & & 2 & 6 \\
\hline Comunicación social & 1 & 1 & 1 & & & & & 3 \\
\hline Contaduría pública & 1 & & & & & & 1 & 2 \\
\hline Cultura física, deporte y recreación & & 1 & & & 1 & & & 2 \\
\hline Derecho & 1 & 2 & 3 & & & & 3 & 9 \\
\hline Diseño gráfico & 1 & & & & & & & 1 \\
\hline Economía & & 1 & & & & & & 1 \\
\hline Estadística & & & & & & & 1 & 1 \\
\hline Gobierno y relaciones internacionales & & & & & 1 & & & 1 \\
\hline Ingeniería civil & & 2 & 1 & & & & & 3 \\
\hline Ingeniería de telecomunicaciones & 1 & & 1 & & & & & 2 \\
\hline Ingeniería electrónica & & & & 1 & & & & 1 \\
\hline Ingeniería industrial & & & 1 & & 1 & & & 2 \\
\hline Ingeniería mecánica & & & & & 1 & & & 1 \\
\hline Licenciatura en filosofía y lengua castellana & & & & & 1 & & & 1 \\
\hline Mercadeo & 1 & & & 1 & & & & 2 \\
\hline Negocios internacionales & & & & 1 & & 1 & 1 & 3 \\
\hline Sociología & 1 & & & & 1 & & & 2 \\
\hline Teología & 1 & & & & & & & 1 \\
\hline Total general & 9 & 7 & 8 & 3 & 8 & $\mathbf{1}$ & 8 & 44 \\
\hline
\end{tabular}

Fuente: elaboración propia.

Análisis

ISSN: 0120-8454 | e-ISSN: 2145-9169 | DOI: https://doi.org/10.15332/21459169

Vol. 53 N.o 98 | enero-junio de 2021 
Por otra parte, en las entrevistas realizadas a los cuatro estudiantes, el docente y el administrativo de la USTA-Bogotá, se pudo evidenciar que el concepto de afrodescendiente es para ellos un concepto universal, en el cual se incluyen no solo las características fisiológicas como el color de la piel, sino también el territorio en el que se nació, las costumbres, los rituales, etc. En este sentido, tener descendencia de africanos y atender a prácticas culturales heredadas de los individuos pertenecientes a esta comunidad, son actos y circunstancias propias de una persona que se identifique como afrodescendiente. Así las cosas, a la pregunta por “¿qué entiende por afrodescendiente?”, un estudiante manifestó: “La definición que encuentro yo de afrodescendiente o una persona afrodescendiente es descendiente de África que recoge ciertas características culturales, étnicas...” (estudiante 2, comunicación personal, octubre de 2019). Otro de ellos afirmó:

Sí, en primera medida y más allá de un tema de un color de piel, lo siento así por el tema cultural, me gusta la música de la región pacífica, que es la región de donde yo soy, que es una región con alto contenido de personas afrodescendientes, por el tema de la relación que tengo con las personas y pues más allá de un tema de explicación, por un tema de identidad que sí siento que me identifico. (Estudiante 3, comunicación personal, octubre de 2019)

Paralelamente a lo expresado por los estudiantes, el docente manifestó: "Las características fisiológicas no son suficientes para determinar la identidad... Lo afro va más allá del color de la piel, tiene que ver con sus creencias con una manera de entender y relacionarse con el entorno y consigo mismo y con los otros" (docente, comunicación personal, octubre de 2019).

Por su parte, el administrativo manifestó: "Nos sometemos al criterio nacional, o sea hay un acuerdo, creo que es un acuerdo, es que no me 
acuerdo, que dice cuáles son los estudiantes afrodescendientes y para eso hay organizaciones que lo certifican" (funcionario administrativo, comunicación personal, octubre de 2019).

A partir de estas respuestas, se evidencia una coincidencia entre las comprensiones de los estudiantes y el docente, mientras que en la entrevista del administrativo, se evidencia poca exactitud en la comprensión más compleja que se tiene de la identidad afrodescendiente. Se evidencia allí la necesidad de que surjan espacios de intercambio, que permitan, tanto con este como con los demás grupos étnicos, comprender la amplitud de características que reúne la identidad para las comunidades y generar así estrategias que respondan a esta comprensión.

\section{Discriminación racial-racismo}

A pesar de que el racismo (como prejuicio), ya sea por el color de piel, el acento, la forma de hablar e incluso las características del cabello, es una situación que podría parecer superada, inexistente u obsoleta, en las entrevistas realizadas a los estudiantes se evidenció que dos de los cuatro entrevistados refirieron haber sido víctimas de racismo prejuicio, pues experimentaron eventos de rechazo, burlas o comentarios o acciones prejuiciosas por sus características fenotípicas y culturales. En este sentido, la Corte Constitucional indicó lo siguiente:

[...] el trato desigual e injustificado que, por lo común, se presenta en el lenguaje de las normas o en las prácticas institucionales o sociales, de forma generalizada, hasta confundirse con la institucionalidad misma, o con el modo de vida de la comunidad, siendo contrario a los valores constitucionales de la dignidad humana y la igualdad, por imponer una carga, no exigible jurídica ni moralmente, a la persona. (Corte Constitucional, Sentencia T- 131/o6, 2006) 
En las entrevistas con los estudiantes afrodescendientes se lograron identificar prácticas de racismo (prejuicio) en algunas interacciones que han tenido actualmente en la USTA. Ese es el caso de una estudiante de la facultad de Derecho:

Estudiante 3: En mi caso, sí he sufrido varios escenarios de discriminación en la Universidad. Fue en primer semestre cuando inicié mi carrera y, a lo largo de este, estuve presentando momentos en los que mucha gente no estaba conmigo, duraba dos o 10 minutos en un grupo y me tenía que salir por la alta presión social por parte de cada uno de los integrantes de estos grupos.

Entrevistadora: ¿Y qué se hizo al respecto?

Estudiante 3: Pues yo sufrí, lloré y diferentes situaciones... Lo llevé a Decanatura, lo llevé a donde el Secretario de División de la Facultad y no hicieron nada al respecto... Otro momento pasó cuando un profesor, más o menos en cuarto o quinto semestre, dijo: "Yo tengo un familiar afro y no quiero que le hagan ningún tipo de vulneración a esta estudiante o a las personas que se encontraban dentro del aula de clases...” ¿Y qué pasó al respecto? Toda la continuación del semestre con ese profesor fue alta presión social. ¿Y quiere respeto? Yo hablaba y no me dejaba. Entonces no sé qué tipo de respeto están promoviendo en esta Universidad.

(Estudiante 3, comunicación personal, octubre de 2019)

Este relato revela no solo el impacto socioemocional que vivió la estudiante a partir de estos comentarios, que se dan en la esfera íntima del aula, sino también la ausencia de un canal institucional para denunciar y acompañar estos eventos, en aras de prevenir en un futuro situaciones de discriminación racial con implicaciones jurídicas.

El segundo estudiante manifestó lo siguiente: 
En una clase, un profesor dijo que la protección de la que estábamos hablando era hecha para personas de ciertos grupos y entre esos hablaba de las comunidades negras o afrodescendientes, y decía que del mismo modo que una persona era discriminada en Bogotá, una persona de Bogotá era discriminada en Cartagena o en un lugar donde la mayoría de la población fuera afrodescendiente. Yo le contesté que no estaba de acuerdo porque se debía tener en cuenta un contexto histórico y él me respondió que había cosas que se debían superar y dejar ahí. Entonces no trascendió más, yo tampoco dije más nada, pero sí fue un momento en el que sentí la discriminación. (Estudiante 2, comunicación personal, octubre de 2019)

Tanto este relato como el anterior evidencian la falta de comprensión que algunos docentes tienen sobre lo que significa la discriminación racial. Como se evidencia, las reivindicaciones no pueden ser trabajo activo solo del estudiante y se dificultan en espacios donde hay cierto poder, como un salón de clases donde predomina la posición del docente o de los estudiantes que no pertenecen a una comunidad étnica históricamente vulnerada. Se dificulta lograr nuevas comprensiones por parte de la comunidad educativa y el estudiante se puede estar viendo enfrentado a una revictimización.

En consonancia con el testimonio anterior, la respuesta del administrativo revela que sí atendió un caso de discriminación en la USTA, pero fue a causa de la actitud del estudiante:

[A él le daba] "vergüenza" ser negro. Estoy tratando de recordar un estudiante... En ese caso él mismo se excluyó: no se ubicaba dentro del grupo y tuvimos que hacerle acompañamiento, porque él no se aceptaba, le daba pena ser negro. (Funcionario administrativo, comunicación personal, octubre de 2019) 
En este caso, se le está atribuyendo la responsabilidad de la exclusión al estudiante por medio del endorracismo, es decir, afirmar que hubo un autorrechazo por una condición étnica y racial. En respuesta a esa misma línea de comportamientos, se consideró oportuno brindarle un acompañamiento que solo se enfocara en su comportamiento y no en el de sus demás compañeros. En este sentido, es importante resaltar que, si bien es importante el acompañamiento individual, en estos casos es necesario comprender que, cuando se presenta una "autoexclusión”, seguramente hubo una exclusión externa previa a este evento, y ese es un elemento clave para ampliar el espectro de acompañamiento.

Por otro lado, en las entrevistas, los estudiantes expresaron que no tienen conocimiento de la existencia de políticas de inclusión ni de un protocolo de atención al que puedan acudir ante la presencia de escenarios de racismo o discriminación por razones étnicas, así como tampoco acciones en torno al acompañamiento estudiantil para estudiantes afrodescendientes. El docente y el administrativo de la USTA corroboran esta información: En la USTA Bogotá no existe actualmente una política de inclusión ni una ruta para el manejo de situaciones de discriminación racial. Se espera que bajo el marco de las políticas de inclusión puedan inscribirse este tipo de estrategias.

En este aspecto es importante señalar que, si bien las personas no afrodescendientes no evidencian este tipo de agresión, y por eso mismo consideran que no existe, la vivencia de las personas afro es completamente diferente. En este sentido, es importante tener en cuenta la percepción de esta comunidad, pues, de lo contrario, se termina invisibilizando esta problemática desde las narrativas de las personas no afrodescendientes. 


\section{Inclusión}

Respecto a la participación de los estudiantes afrodescendientes en la construcción de las políticas de inclusión, se evidencia que este grupo étnico no fue convocado de manera explícita para la construcción de estas:

Bueno, los estudiantes... ha habido varios procesos de participación. Se hicieron varios grupos focales de estudiantes, docentes, directivos y administrativos, y en los grupos focalizados se hizo una convocatoria a algunos estudiantes que consideramos. No recuerdo si haya participado algún afrodescendiente, no recuerdo... Ah sí, pero ella estaba en doble condición, víctima del conflicto armado y afrodescendiente. (Funcionario administrativo, comunicación personal, octubre de 2019)

Sin embargo, se evidencia la disponibilidad y cuestionamiento por parte del funcionario administrativo para comprender a fondo la situación de los estudiantes afrodescendientes en la USTA Bogotá:

Pero, por ejemplo, ¿cuáles son las necesidades de un estudiante afrodescendiente?, ¿¿cuándo nos hemos preguntado qué es lo que necesita él?, ¿̇sí? O sea, constitucionalmente son de los grupos priorizados, constitucionalmente nos dice que esas comunidades deben estar priorizadas. ¿Y cuándo constitucionalmente nos hemos preguntado cuál es la prioridad, por qué es prioritario, qué es lo que necesita y por qué es una comunidad de prioridades? No lo hemos hecho, entonces el paso a seguir es preguntar a estos grupos qué es lo que verdaderamente necesitan institucionalmente a nivel administrativo, académico, socioeconómico, institucional. Ahí ya la política aterriza. (Funcionario administrativo, comunicación personal, octubre de 2019)

El siguiente paso consiste en comprender que la inclusión va más allá de cubrir necesidades. Para el caso de las comunidades étnicas, se trata de abrir espacios en la Universidad para la interacción con los saberes ancestrales de estos grupos. Se trata de un diálogo en el que la comunidad 
universitaria no afrodescendiente pueda interactuar con estos saberes y hacer a la vez una legitimación de su importancia histórica en la construcción del país.

Adicional a esto, a partir de las entrevistas realizadas en este estudio, se evidencia la necesidad de propiciar un espacio de encuentro de estudiantes afrodescendientes de la USTA Bogotá, la justificación de este espacio es claramente descrita por uno de los estudiantes:

Reunir a todos los afros de la USTA y poder dar información del apoyo que se quiere brindar desde la Universidad, como somos una población minoría en la Universidad sería bueno hacer esos campos de todos unirnos y saber todas las cosas que se pueden hacer hacia los afros. Además, ese acompañamiento debería ser adicionado en cuanto a un acompañamiento histórico, decir: bueno ċqué ha pasado con la comunidad afro y su trayectoria? (Estudiante 3, comunicación personal, octubre de 2019)

\section{Inclusión. Acciones afirmativas}

Se revisaron tres documentos institucionales: Documento marco de desarrollo integral del estudiante (USTA, 2015), Reglamento de auxilios y descuentos educativos (USTA, 2019) y el Informe de gestión de la Rectoría General, 2015-2018 (USTA, 2018), por considerarlos de importancia en el panorama actual de los procesos de inclusión de la USTA.

En dos de los tres documentos - Documento marco de desarrollo integral del estudiante (USTA, 2015) y el Informe de gestión de la rectoría general 2015-2018 (USTA, 2018) - no se encontraron referencias explícitas a la comunidad afrodescendiente. En este sentido, no es posible encontrar menciones orientadas al tratamiento de las situaciones de racismo, discriminación racial o acciones afirmativas para este grupo de población. 
En relación con el Reglamento de auxilios y descuentos educativos (USTA, 2019), se presenta la definición de "comunidades afrodescendientes", relacionada en la introducción. Asimismo, se define un auxilio del $20 \%$ para esta población, previo cumplimiento de requisitos administrativos y de la "carta de presentación del estudiante como integrante del resguardo o comunidad a la cual pertenece". Sin embargo, como lo mencionará un estudiante más adelante, esta certificación ha llegado a tener un costo similar al del descuento, por lo cual los estudiantes no la solicitan ni acceden a este beneficio.

\section{Discusión}

La comprensión de identidad afrodescendiente en interior de la USTA es una tarea que se debe ir complejizando. Los hallazgos presentados en este estudio evidencian una falencia habitual en las IES: la desarticulación entre las discusiones teóricas, las luchas comunitarias y las decisiones administrativas. Esto puede hacerse evidente en la denominación de las comunidades afrodescendientes como "negritudes" en el sistema académico SAC, que hace referencia solo a características fenotípicas y desconoce el conjunto de elementos que definen la identidad afrodescendiente, así como las discusiones teóricas contemporáneas en relación con este aspecto. Sin embargo, esto no es algo que solo suceda en la USTA, también sucede en la Universidad del Estado de Río de Janeiro, donde el sistema de cuotas mínimas está destinado para estudiantes autodenominados negros.

En este sentido, es pertinente que haya una articulación entre las construcciones que se dan desde la academia y las mismas comunidades afrodescendientes, con las políticas y lineamientos, de manera que se materialicen claramente en documentos orientadores que presenten disposiciones concretas de carácter institucional. 
Asimismo, a partir de las entrevistas realizadas, se evidencia una diferencia entre la comprensión del concepto de identidad afrodescendiente explicitado por el funcionario administrativo y lo planteado por los estudiantes, el docente y la misma teoría, en los que se reivindica la importancia histórica y la complejidad cultural de este grupo étnico que se deriva de una reivindicación de derechos (Hopenhayn et ál., 2006). Lo anterior reafirma la necesidad de vincular la academia con los procesos administrativos, de modo que se logren discursos transversales que favorezcan la incorporación de estos principios en las decisiones y documentos de inclusión y acompañamiento estudiantil en la USTA Bogotá.

Ahora bien, al hablar de inclusión para comunidades afrodescendientes en la USTA Bogotá, se identifica la incorporación de acciones afirmativas en el caso de los descuentos de matrícula. En los hallazgos de las seis IES latinoamericanas revisadas, se encuentra que tres de ellas se acogen como única forma de acción afirmativa al sistema de cuotas mínimas y las otras tres implementaron diversas formas y manifestaciones de acciones afirmativas en sus instituciones. Lo anterior se plantea en la tabla 3.

Tabla 3. Comparativa de inclusión por universidades, en acciones afirmativas

\begin{tabular}{|c|c|c|}
\hline IES latinoamericanas & $\begin{array}{c}\text { Cuota de } \\
\text { cupos/descuentos }\end{array}$ & Otras acciones afirmativas \\
\hline $\begin{array}{l}\text { Caso Chile: Universidad } \\
\text { de la Frontera }\end{array}$ & & $\begin{array}{l}\text { Se implementaron acciones afirmativas por } \\
\text { medio del programa RUPU. }\end{array}$ \\
\hline Caso Perú & & $\begin{array}{l}\text { Se constituyeron dos universidades } \\
\text { indígenas, además de otras bilingües en las } \\
\text { que se imparten clases en castellano y } \\
\text { determinado dialecto indígena. }\end{array}$ \\
\hline $\begin{array}{l}\text { Caso Brasil: Universidad } \\
\text { del Estado de Río de } \\
\text { Janeiro }\end{array}$ & $\begin{array}{l}\text { Sistema de cuotas } \\
\text { mínimas }\end{array}$ & \\
\hline $\begin{array}{l}\text { Caso Colombia: } \\
\text { Universidad del Caribe }\end{array}$ & $\begin{array}{l}\text { Sistema de cuotas } \\
\text { mínimas }\end{array}$ & Otras acciones afirmativas \\
\hline
\end{tabular}




\begin{tabular}{|l|l|l|}
\hline IES latinoamericanas & $\begin{array}{c}\text { Cuota de } \\
\text { cupos/descuentos }\end{array}$ & Otras acciones afirmativas \\
\hline $\begin{array}{l}\text { Caso Colombia: } \\
\text { Universidad del Valle }\end{array}$ & $\begin{array}{l}\text { Sistema de cuotas } \\
\text { mínimas }\end{array}$ \\
\hline Caso Colombia: USTA & $\begin{array}{l}\text { Descuentos en el } \\
\text { porcentaje de la } \\
\text { matrícula }\end{array}$ \\
\hline
\end{tabular}

Fuente: elaboración propia.

Estas acciones tienen un papel relevante ya que, según Alfaro (2007) pretenden realizar una reivindicación histórica con miras a construir una sociedad menos desigual y un orden justo. Además de esto, toda acción afirmativa contiene una carga y obligación razonable que debe ser asumida por la sociedad en conjunto y que, en este caso, por la Universidad, de manera que cumpla al trato equitativo a la comunidad afrodescendiente. Esta alternativa da cuenta de un primer avance en el reconocimiento y compromiso de la USTA por cerrar la brecha histórica de exclusión.

Sin embargo, los hechos de racismo en la USTA narrados por los estudiantes en las entrevistas muestran que el sistema de cuotas desarrollado por la Universidad como única acción afirmativa, y representada como política de inclusión, no es suficiente para incluir de forma efectiva a la comunidad afrodescendiente de la USTA y tampoco es el mecanismo pertinente para abordar los hechos discriminatorios narrados con el fin de ser detenidos. Todo ello hace evidente la necesidad de construir una ruta de atención para situaciones de racismo y discriminación racial. Esto favorecerá que la Universidad declare de manera explícita un respaldo hacia la comunidad afrodescendiente y, en esa misma medida, la comunidad denuncie y se acoja a un acompañamiento que, está claro, no sería solo para los sujetos involucrados, sino para la comunidad entera y, finalmente, permitiría enfrentar la dificultad del subregistro e invisibilización de esta 
problemática, pues, al no tener canales claros de atención, no se reportan los casos y se cree que la situación no está ocurriendo.

Esta identificación y acompañamiento a las situaciones de racismo y discriminación racial son un elemento favorable no solo para la población afrodescendiente, sino para toda la comunidad educativa. Ampliar la mirada que se tiene desde la Universidad a las comunidades afrodescendientes redunda no solo en la construcción de una sociedad más equitativa, sino que permite a la Universidad diversificar sus miradas. Las comunidades afrodescendientes y sus individuos son portadores de una tradición histórica y cultural que es preciso incorporar en la Universidad, a fin de que la inclusión se traduzca también en la articulación con sus significados, formas de vida y apuestas políticas. Esto implicaría, a su vez, la generación y fortalecimiento de espacios y jornadas académicas, destinadas a la socialización de estos aspectos:

Pensar en los aportes de las culturas afroamericanas a la América Latina actual significa hacer que muchos símbolos culturales y estrategias de lucha política para las comunidades negras de Iberoamérica, como el cimarronaje y la afrocentricidad, decantados a lo largo de cinco siglos, sean ahora finalmente incorporados a la gramática general del pensamiento iberoamericano. (Carvalho, 2009, p. 27)

En este sentido, es importante resaltar que un plan de inclusión para los grupos étnicos beneficia no solo a quien pertenece a un grupo, sino a la comunidad universitaria en su globalidad, pues permite a todos los actores interactuar y comprender el aporte que ha tenido la comunidad a nivel histórico y académico, así como comprender sus comportamientos y lenguajes, creencias y tradiciones, y cómo han sido relevantes en la construcción del país. No contar con estrategias de inclusión perjudica no solo a los sujetos afrodescendientes sino a la comunidad entera, al perder 
la posibilidad de recuperar esos saberes e incluirlos en el tejido de la comunidad universitaria.

De allí, resulta la urgencia abrir espacios de participación, claramente determinados para este y otros grupos étnicos, a fin de que ellos mismos tengan un lugar en la construcción de las acciones de inclusión y acompañamiento, y puedan explicitar sus aportes y requerimientos frente a la Universidad. Esto favorecerá además una comprensión adecuada de las características de la población afrodescendiente de la USTA-Bogotá. Ahora bien, dado que las políticas de inclusión en las IES están orientadas a toda la comunidad educativa, en un próximo estudio valdría la pena ampliar la exploración realizada en este ejercicio investigativo a personas afrodescendientes pertenecientes a la USTA Bogotá como directivos, administrativos, docentes, y personal de apoyo. Tal como lo manifiesta la administrativa entrevistada: "La política de inclusión no solo debe ir a los estudiantes; también a los docentes, a los administrativos, a los directivos" (funcionario administrativo, comunicación personal, octubre de 2019). En conclusión, puede afirmarse que las universidades latinoamericanas abordadas y, en particular, la USTA Bogotá se enfrentan en la actualidad al reto de articular la trayectoria jurisprudencial, académica y comunitaria con los documentos y políticas que orientan la interacción con la comunidad afrodescendiente. Con esto, se garantiza una inclusión que favorezca a toda la comunidad y se oriente a la desnaturalización y eliminación de los escenarios de racismo y discriminación racial.

\section{Referencias}

Arroyo, M. (2017). La educación intercultural: un camino hacia la inclusión educativa.

Educación Inclusiva, 6(2), 144-159. 
Benavides, M. O. y Gómez-Restrepo, C. (2005). Métodos en investigación cualitativa: triangulación. Revista Colombiana de Psiquiatría, 34(1),118-124. http://www.scielo.org.co/scielo.php?script=sci $\operatorname{arttext\& pid=So034-}$ 74502005000100008\&lng $=$ en\&tlng $=\mathrm{e}$

Carvalho, J. J. (2009). Cimarronaje y afrocentricidad: los aportes de las culturas afroamericanas a la América Latina contemporánea. Pensamiento Iberoamericano, 4, 25-48. https://dialnet.unirioja.es/servlet/articulo?codigo $=7079769$

Constitución Política de Colombia. (1991). Gaceta Constitucional, 116. https://www.corteconstitucional.gov.co/inicio/Constitucion\%20politica\%20de\%2 oColombia.pdf

Corte Constitucional. (2016, 5 de diciembre). Sentencia T-68o/16 (Jorge Iván Palacio, M. P.). https://bit.ly/3eaGe2G

Corte Constitucional. (2006, 23 de febrero). Sentencia T-131/o6 (Alfredo Beltrán Sierra, M. P.). https://www.corteconstitucional.gov.co/relatoria/2006/T-131-06.htm

Corte Constitucional. (2010, 21 de abril). Sentencia C-293/1o (Nilson Pinilla Pinilla, M P.). https://bit.ly/2O5tYFP

Corte Constitucional. (2017, 22 de febrero). Sentencia C-115-2017 (Alejandro Linares Cantillo, M. P.). https://www.corteconstitucional.gov.co/relatoria/2017/C-11517.htm

Corte Constitucional. (2003, 21 de octubre). Sentencia C-964/o3 (Álvaro Tafur Galvis, M. P.). https://bit.ly/2PAgFha

Freire, G., Díaz-Bonilla, C., Schwartz, S., Soler López, J. y Carbonari F. (2018). Afrodescendientes en Latinoamérica: hacia un marco de inclusión. Grupo Banco Mundial.

https://openknowledge.worldbank.org/bitstream/handle/10986/30201/1292987-8-2018-17-30-51AfrodescendientesenLatinoamerica.pdf? sequence $=5$ \&isAllowed $=\mathrm{y}$

Hermida, J. F. (2007). Acciones afirmativas e inclusión educacional en Brasil. En Escenarios mundiales de la educación superior. Análisis global y estudios de casos (pp. 193-228). CLACSO. http://biblioteca.clacso.edu.ar/clacso/formacionvirtual/20100719075611/o7Hermida.pdf 
Hopenhayn, M., Bello, A. y Miranda, F. (2006). Los pueblos indígenas y afrodescendientes ante el nuevo milenio. Cepal. https://repositorio.cepal.org/handle/11362/6123

Congreso de la República de Colombia. (1993, 27 de agosto). Ley 7o. Por la cual se desarrolla el artículo transitorio 55 de la Constitución Política. Diario Oficial n. ${ }^{\circ}$ 41.013. http://www.secretariasenado.gov.co/senado/basedoc/ley_oo70_1993.html\#: :tex t=La\%20presente\%20ley\%20tiene\%20por,con\%20lo\%20dispuesto\%20en\%20los

Morón-Cárdenas, J. A., Pedrozo-Martínez, A. M. y Torres-Cabrera, M. A. (2013). Acciones afirmativas como mecanismo de inclusión social en la educación superior pública: el caso de la Región Caribe Colombiana. Clío América, 7(14), 110-119. https://revistas.unimagdalena.edu.co/index.php/clioamerica/article/view/756

Sanborn, C. y Arrieta, A. (2011). Universidad y acción afirmativa: balance y agenda pendiente. Centro de Investigación de la Universidad del Pacífico. https://repositorio.up.edu.pe/bitstream/handle/11354/376/DD1116.pdf?sequence =1\&isAllowed $=\mathrm{y}$

Segato, R. (2007). Racismo, discriminación y acciones afirmativas: herramientas conceptuales. En Alfaro, S. González, M. Mujica, L. Segato, R. Villasante, M. Educar en ciudadanía intercultural (pp. 63-91). Pontificia Universidad Católica del Perú. Fondo Editorial.

http://repositorio.pucp.edu.pe/index/handle/123456789/53738

Septien, R. C. y Bidaseca, K. (eds.). (2017). Más allá del decenio de los pueblos afrodescendientes. CIPS, Centro de Investigaciones Psicológicas y Sociológicas. http://www.clacso.org.ar/librerialatinoamericana/libro detalle.php?id libro=1289\&orden=nro orden\&pageNum $\underline{\text { rs libros }=0 \& \text { totalRows } r \text { libros }=1236 \& \text { orden }=\text { nro orden }}$

Universidad de La Frontera. (2019). Programa de apoyo académico para estudiantes Mapuche. Universidad de la Frontera. https://www2.ufro.cl/servicios/menu_cont.php?cod_menu=2\&cod_pagina=2\&sit $\equiv 30$ 
Universidad del Valle. (2003, 16 de octubre). Resolución n. ${ }^{\circ}$ o97. Por la cual se crea la Condición de Excepción comunidades afrocolombianas para el ingreso a los Programas Académicos de Pregrado.

http://proxse16.univalle.edu.co/ secretariageneral/consejoacademico/resoluciones/2003/CA-097-CondiciondeExcepcion-Negritudes.pdf

Universidad Santo Tomás. (2018). Informe de gestión - Rectoría General 2015-2018. Universidad Santo Tomás.

http://planeacion.usta.edu.co/images/documentos/Informe RECTOR GENERA

L Nov 18.pdf

Universidad Santo Tomás. (2019). Reglamento de auxilios y descuentos educativos

USTA. https://sindicatura.usta.edu.co/index.php/recursos/reglamento-deauxilios-y-descuentos-educativos 